\title{
Pulse, polarization and topology shaping of polariton fluids
}

\author{
Lorenzo Dominici ${ }^{\mathrm{a}}$, David Colas ${ }^{\mathrm{b}}$, Stefano Donati ${ }^{\mathrm{b}}$, Galbadrakh Dagvadorj $^{\mathrm{c}}$, \\ Antonio Gianfrate ${ }^{\mathrm{a}}$, Carlos Sánchez Muñoz ${ }^{\mathrm{b}}$, Dario Ballarini ${ }^{\mathrm{a}}$, Milena De Giorgi ${ }^{\mathrm{a}}$, \\ Giuseppe Gigli ${ }^{\mathrm{a}}$, Marzena H. Szymańska ${ }^{\mathrm{d}}$, Fabrice P. Laussy ${ }^{\mathrm{b}}$, and Daniele Sanvitto ${ }^{\mathrm{a}}$ \\ ${ }^{a}$ CNR NANOTEC, Istituto di Nanotecnologia, Via Monteroni, 73100 Lecce, Italy \\ ${ }^{\text {b}}$ Departamento de Física Teórica de la Materia Condensada, UAM, 28049 Madrid, Spain \\ ${ }^{\mathrm{c}}$ Department of Physics, University of Warwick, CV47AL Coventry, UK \\ ${ }^{\mathrm{d}}$ Department of Physics and Astronomy, University College London, WC1E6BT London, UK
}

\begin{abstract}
Here we present different approaches to ultrafast pulse and polarization shaping, based on a "quantum fluid" platform of polaritons. Indeed we exploit the normal modes of two dimensional polariton fluids made of strong coupled quantum well excitons and microcavity photons, by rooting different polarization and topological states into their sub-picosecond Rabi oscillations. Coherent control of two resonant excitation pulses allows us to prepare the desired state of the polariton, taking benefit from its four-component features given by the combination of the two normal modes with the two degrees of polarization. An ultrafast imaging based on the digital off-axis holography technique is implemented to study the polariton complex wavefunction with time and space resolution. We show in order coherent control of the polariton state on the Bloch sphere, an ultrafast polarization sweeping of the Poincaré sphere, and the dynamical twist of full Poincaré states such as the skyrmion on the sphere itself. Finally, we realize a new kind of ultrafast swirling vortices by adding the angular momentum degree of freedom to the two-pulse scheme. These oscillating topology states are characterized by one or more inner phase singularities tubes which spirals around the axis of propagation. The mechanism is devised in the splitting of the vortex into the upper and lower polaritons, resulting in an oscillatory exchange of energy and angular momentum and in the emitted time and space structured photonic packets.
\end{abstract}

Keywords: Rabi oscillations, coherent control, polarization shaping, quantum vortices, polaritons

\section{INTRODUCTION}

We make use of microcavity polaritons, ${ }^{1}$ a "quantum fluid" platform constituted by bosonic hybrid quasiparticles of strongly coupled excitons and photons fields, in order to achieve different kind of intensity, polarization and topology shaping. Due to the photonic outcoupling of the polaritons, which makes possible their resonant excitation and detection, such time and space structured-field features are tranferred to the emitted optical pulses too. The realization of exciton polariton condensates in semiconductor microcavities ${ }^{2,3}$ has paved the way for a prolific series of studies into quantum hydrodynamics in two-dimensional systems. ${ }^{4-12}$ Microcavity polaritons are intriguing systems for the study of topological excitations in nonequilibrium interacting superfluids, also thanks to rich spinorial patterns and polarization splitting terms leading to typical spin-orbit coupling. ${ }^{13-15}$ In very recent works, ${ }^{16,17}$ thanks to significant progress in both the quality of the structures and in the laboratory state of the art, we have been able to both observe and control the microcavity polariton Rabi dynamics. We can span from Rabi oscillating configurations to eigenstate superpositions, and control them by multiple optical pulses that can amplify or switch states. Moreover, by exploting the spin degree of freedom, we have been able to tranfer the intensity oscillations into polarization oscillations, thus making such systems even more suitable for polarization shaping engineering and applications. Space structured topology such as full Poincaré skyrmion beams have be imprinted too and their reshaping described in the polarization space. ${ }^{18}$ The most recent of our experiments and models are hence pushed further on, by empowering with the Rabi oscillations the ultrafast topology shaping of quantized vortices, as shown in the last section of this work.

Further author information: (Send correspondence to L.D.)

L.D.: E-mail: lorenzo.dominici@gmail.com, Telephone: +390832 319210

F.P.L.: E-mail: fabrice.laussy@gmail.com 


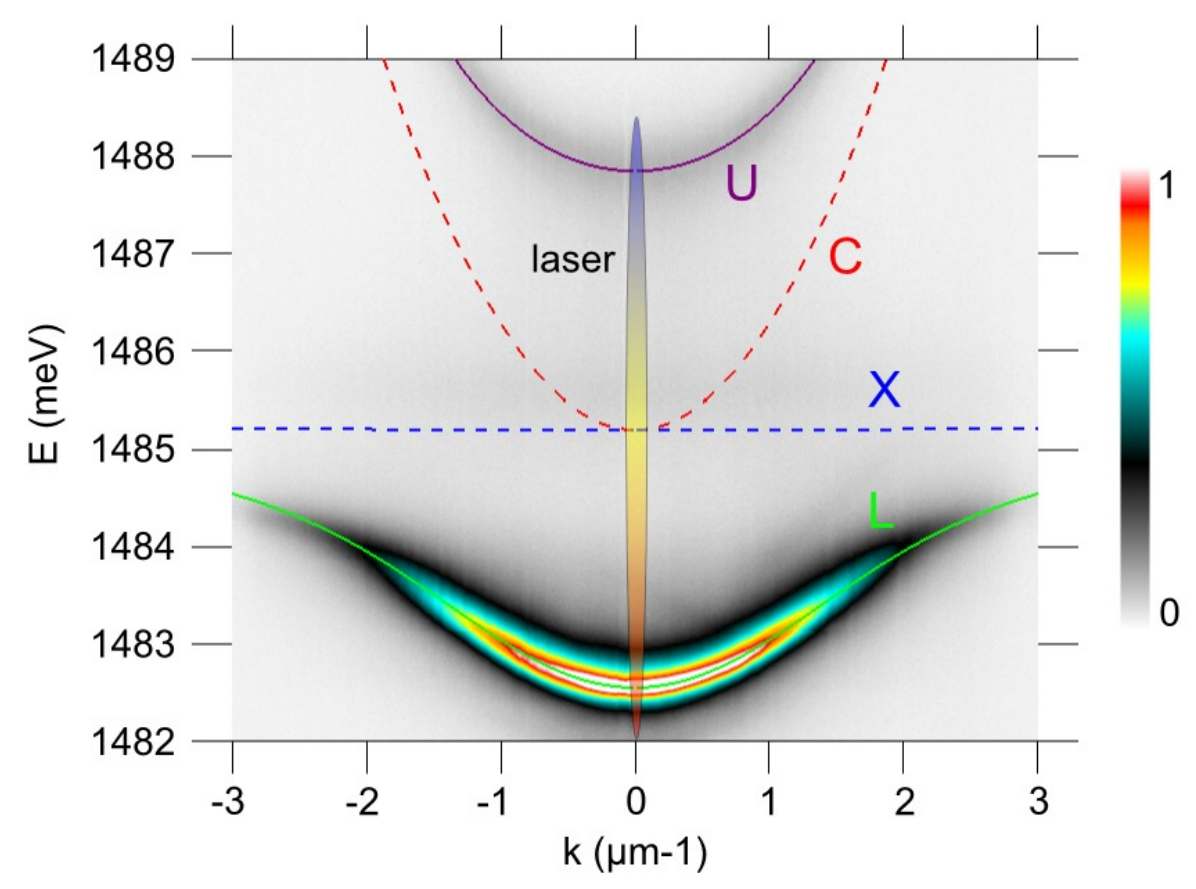

Figure 1. Experimental polariton dispersion. The emission is obtained after off-resonant excitation. The bare modes are denoted as dashed lines, microcavity mode ( $\mathrm{C}$, red) and quantum well exciton (X, blue). The polariton normal modes are denoted as solid lines, UPB (U, violet) and LPB (L, green). A sketch of a resonant femtosecond laser tuned to excite both LPB and UPB branches is shown as a gradient color ellipse at $k=0$. Data readapted from Dominici et al. (2015). ${ }^{5}$

\section{POLARITON RABI OSCILLATIONS AND COHERENT CONTROL}

An important peculiarity of polaritons here is at play, the so called Rabi oscillations. ${ }^{16,17}$ Indeed, a strong coupling of the two bare oscillators, the microcavity photons $(\mathrm{C})$ and the quantum well exciton (X) fields, results also in their splitting into two normal modes of the system. These are the upper (UPB) and lower polariton branch (LPB) modes, which differ in energy and dephasing time (which is, mode bandwidth), and where the two bare fields oscillate with in-phase and phase-opposition, respectively. The simultaneous excitation of the two modes can be perceived as a time beating in their projection upon the photon and exciton basis. A complementary point of view is the continuous transformation of photons into excitons and then back. Rabi oscillations have been known since the early pioneering works on polaritons, but their space resolved ultrafast imaging only recently achieved, and never before exploting the high degree of coherence as here. Typical bare and normal modes of microcavity polaritons are shown in Fig. 1.

Cyclical exchange of excitation between two coupled modes $a$ and $b$ can occur at either the single particle or many particle levels. When this occurs at the single particle level in a quantum two-level system, it provides the ground for a qubit, ${ }^{19}$ which, if it can be further manipulated, opens the possibility to perform quantum information processing. ${ }^{20}$ Such an oscillation is of probability amplitudes and therefore is a strongly quantum mechanical phenomenon, that involves maximally entangled states:

$$
|\Psi(t)\rangle=\alpha(t)\left|1_{a}, 0_{b}\right\rangle+\beta(t)\left|0_{a}, 1_{b}\right\rangle .
$$

The same physics also holds, not at the quantum level, but with coherent states of the fields, a situation known in the literature as implementing an "optical atom" ${ }^{21}$ or a "classical two-level system". ${ }^{22}$ The oscillation is then more properly qualified as "normal mode coupling" 23,24 as it is now between the fields themselves:

$$
|\psi(t)\rangle=|\alpha(t)\rangle|\beta(t)\rangle
$$


rather than their probability amplitudes. The terminology of Rabi oscillations is however used also in the classical case, ${ }^{25,26}$ such for example the purpose to realize classical bits, "cebit". ${ }^{27}$ or other classical counterparts of the quantum version. ${ }^{28}$ As a futher example, classical two-level systems ${ }^{29}$ were recently pursued in the rising field of nanomechanical optics. ${ }^{22,30}$ In our case, the polariton system ${ }^{31}$ provides hence a good platform to implement two-level dynamics at both the quantum ${ }^{32}$ and classical level. ${ }^{33}$ As said, the polariton nature itself is that of a two-level system, giving the strong coupling regime between the cavity photon and a semiconductor exciton, also named bare oscillators. In the specific, two-dimensional microcavities embedding inorganic quantum wells $(\mathrm{QWs})^{34}$ have attracted attention for their ability to reach the regime of Bose-Einstein condensation ${ }^{35}$ and superfluidity, ${ }^{11,36}$ with a plethora of collective wavefunction phenomena hydrodynamics including coherence, quantized vorticity, strong nonlinearities and real-space pattern formation. ${ }^{4,5,8,37,38}$ On the application side, the most intriguing perspectives involve schemes and demonstrations for new polariton lasers ${ }^{39,40}$ and all-optical transistors and logical operations. ${ }^{41}$ Pioneering attempts to observe the Rabi oscillations at the core of the polariton physics, encountered intrisic difficulties represented, e.g., by their sub-ps time range and reported very few oscillations with orders of magnitude visibility loss each cycle. ${ }^{42}$ The inhomogeneous broadening of excitons, ${ }^{43}$ not taking into account the space dephasing of the space-integrated signals, could provide a qualitative agreement only. Later on, nonlinear pump-probe techniques, ${ }^{44-47}$ achieved a better visibility in the registration of the modes beating but didn't provided the necessary degree for a deeper analysis. Our recent experiments could image the time and spatially resolved oscillations and achieve Rabi piloting by two pulse schemes able to achieve the wished modes superposition including a polarization degree of freedom. Such schemes are of paramount importance ${ }^{48}$ also in light of the possibility to address polaritons at the single particle level. ${ }^{49,50}$

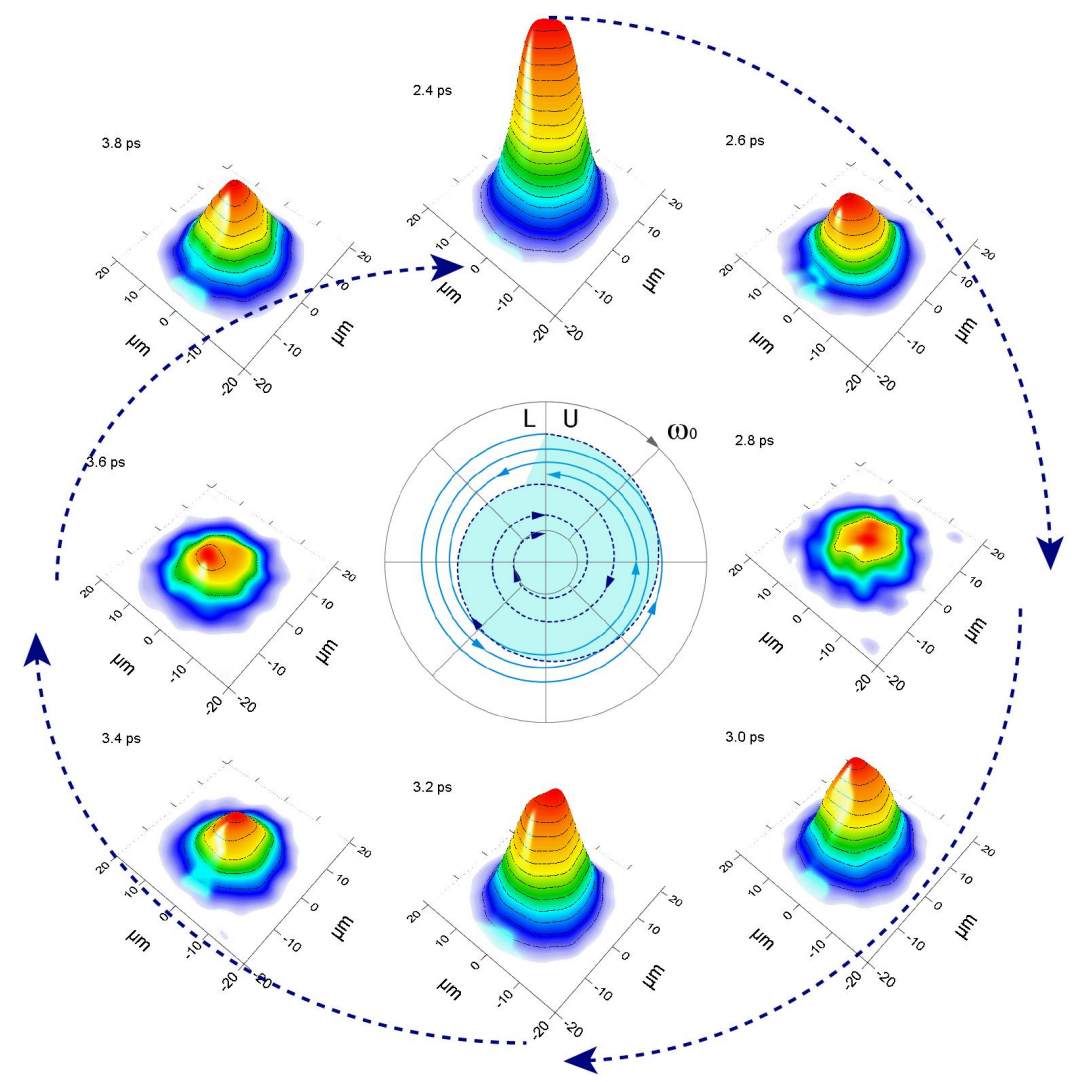

Figure 2. Two Rabi cycles seen through the time and space resolved photonic emission (outer panels) and the scheme of the UPB and LPB modes depiceted in the $\omega_{0}$ rotating frame of reference (inner panel). The time reference is given by the central frequency between the bare modes $\left(\omega_{0}=\frac{\omega_{C}+\omega_{X}}{2}\right)$. 


\subsection{Single pulse Rabi oscillations}

As shown in Fig. 1, the $130 \mathrm{fs}$ long and $8 \mathrm{~nm}$ energy broad laser pulse spreads in energy over the two branches, preparing the system in a bare state and not in an eigenstate, inducing oscillations between its two components. One can see on the outer panels of Fig. 2 a typical experimental observation of the space resolved photonic emission from the microcavity sample. As expected, the photon field arises and vanishes cyclically, during each Rabi cycle. The different dephasing times of the two modes, suggested by the inner scheme of the figure (with different radius of the rotating modes), is leading also to a damping of the oscillations between consecutive cycles. Here the polaritons have quite a large spatial extension, given by the exciting laser, $\approx 10 \mu m$. However, given the system is linear, presents a minimal diffusion and is not imparted by any momentum, the dynamics can be reduced to zero dimension (i.e., the field is homogeneous and with no space reshaping, there is no exploitation of the space degree of freedom). Indeed, the excitation power was set at a low enough density in order to maintain their bosonic properties in the linear regime. We could have thus access to the subpicosecond linear Rabi oscillations through the coherent fraction $\left|\psi_{C}(r, t)\right|^{2}$ of the photonic field.

To this extent, our setup can access the complex wavefunction, i.e., measuring both its amplitude and phase, by holography, a technique of increasing use to image polariton fluids for which both of these informations are of crucial importance. ${ }^{51,52}$ We recourse to a variation known as off-axis digital holography, ${ }^{53}$ which provides highquality results by separation of the diffracted images of an off-axis reference frame and the signal. We adapted it to support ultrafast and tunable multiple-pulse experiments, as shown in Fig. 3. As such, our measurement does not rely on nonlinear detection scheme or interactions, as in previous works, ${ }^{44-46}$ but on interferences only. The photonic field that is accessible experimentally, have been fitted with the optical model developed in detailed in, ${ }^{16}$ and its excitonic counterpart recovered through the polaritonic equations .

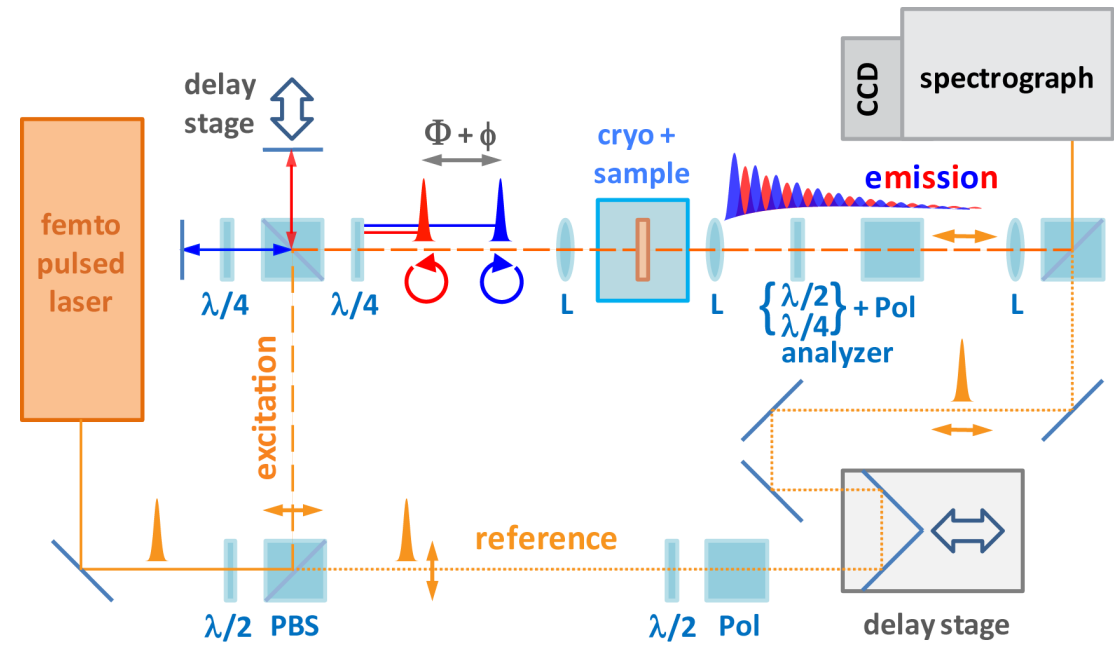

Figure 3. Experimental setup for double pulse coherent control experiments with polarization control and digital off-axis holography imaging. The interference between the device emission and the reference arms is implemented through a Mach-Zehnder configuration, while the two-pulse excitation is achieved in a Michelson configuration.

\subsection{Two-pulse coherent control}

With such an accurate command of the system, we are able to time precisely the arrival of a second pulse and perform a comprehensive coherent control on the coupled dynamics. The Rabi oscillations are triggered by sending a first pulse quite broad in energy that initiates a dominant photon or exciton fraction. One can refine the state by sending a second pulse, similar to the first one, to any desired configurations. The delay of the second photonic pulse can be set according to four different main preparations, depending on the combination of the pulses' relative delay in both the Rabi period order $\Phi$ and optical period order $\phi$. For example, the Rabi antiphase condition is achieved upon coupling the second pulse when the cavity field is empty and the state is fully excitonic. In such case, injecting a second fully photonic pulse in optical (resp. anti-optical) phase with the 
exciton, for instance, creates an LP (resp. UP), as shown in Fig. 4(a) (resp. Fig. 4(b)). The transformation of a bare state, created by the 1st pulse, into a LPB or UPB state when the second pulse arrives, is associated to the switching off of the Rabi oscillations, and the population decreases with the lifetime of the respective mode. In Fig. 4(c), the second pulse is arriving during the photonic phase of the condensate (Rabi in-phase condition), and also in optical phase with the cavity field. This is a case of Rabi oscillations enhancement, where the oscillations cycle is magnified with the second pulse. In the case of Fig. 4(d) instead the second pulse annihilates the field. This is achieved by sending a pulse optically out of phase but in phase with the Rabi oscillations, inducing destructive interferences that cancel the field intensity. All these cases demonstrate the possibility to do coherent control of the strong light-matter coupling dynamics. Such proof of principle fully demonstrated here also suggests that there would be no fundamental difficulty in sending more than two pulses. Interesting perspectives are now opened, notably at the single-particle level to perform quantum information processing.
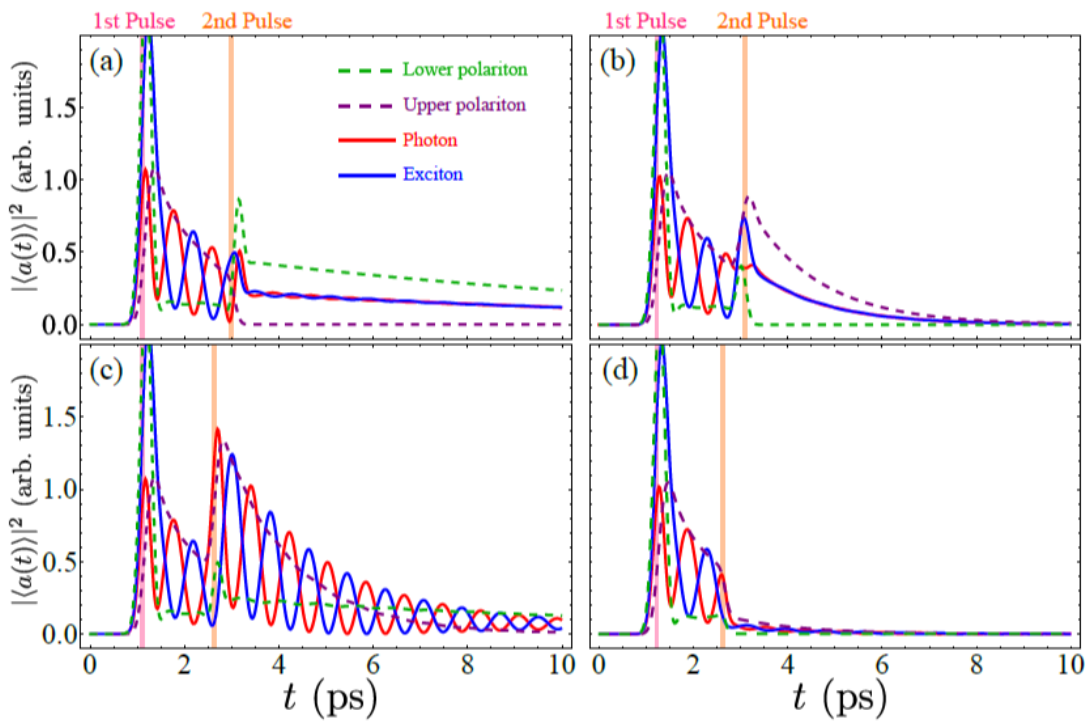

Figure 4. Two-pulse experiment as seen through all the theoretical variables: bare states in solid and eigenstates in dashed lines. The experimentally available variable is the photon field, in red. The four cases correspond to: (a) switching-off of the Rabi oscillation by bringing the state into a LPB condensate, (b) switching-off of the Rabi oscillation by bringing the state into a UPB condensate, (c) enhancement of the oscillations and (d) annihilation of the field. Reprinted with permission from Dominici et al. (2014). ${ }^{16}$ Copyright (2014) by the American Physical Society.

\section{RABI POLARIZATION SHAPING}

We here discuss how to generate new states of light by using this most fundamental of properties from the polaritons, their Rabi oscillations. The realization is based on the proposal to link these two concepts of polarized light by using the oscillating property of the microcavity polaritons. The idea is the following: a light whose polarization is varying in time can be emitted from a microcavity by exciting it with two orthogonally polarized pulses and with a suitable time delay between them. Working with orthogonally polarized pulses ensures that no interactions between the fields of different polarizations will occur. The superposition of the oscillating fields results in the precession of the Stokes vector of the emitted light. Polaritons being particles with finite lifetime, it results in a drift of the polarization from a circle on the Poincaré sphere (whose radius and position can be controlled) to a single point at long time. This leads to the generation of a new kind of polarized light, suitable to cover selected area of the Poincaré sphere, and possibly the whole sphere, in a range of a few picoseconds. The effect has been demonstrated as a proof of principle with microcavity polaritons at the femtosecond time scale in our previous work. It shows the emission of time varying polarized light, that, for instance, can cover a whole 
hemisphere of the Poincaré sphere. But as it relies on the Rabi oscillations, this effect could be thus transposed and obtained with other platforms operating at different time scales (from attoseconds to milliseconds) and exploiting the same property.

\subsection{Polarization Shaping}

The first realization of a shaped pulse produced light whose intensity, momentary frequency and light polarization were varied as a function of time in a timescale of femtoseconds. This was reported by Brixner and Gerber in $2001 .{ }^{54}$ Thereby was opened the era of "polarization pulse shaping" with a huge number of potential applications. Before that, only simple polarization profiles could be achieved by using interferometric combinations of two polarization components. It has been demonstrated by Zhuang et al. (1997) ${ }^{55}$ that a stack of three homogeneous nematic liquid-crystal cells, or Liquid-Crystal display (LCD) could be used as a controller to bring any state of polarization of light from one arbitrary state to another. The idea of Brixner and Gerber was thus to build a device based on a set of many-pixel (256) two-layer LCDs to obtained a time varying polarized light, each pixel changing the state of polarization (SOP). By applying a suitable voltage to the separated pixels, one tune individual frequency intervals throughout the laser spectrum. The phases differences leasing to a complex polarization-modulated laser pulses in the time domain. The desired sequence of voltage modulations, that correspond to the various SOPs, can be computer-controlled with a corresponding algorithm.

The range of applications is potentially huge since the interaction between light and matter is polarization sensitive. This technique was thus used to increase the performances of many applications based on polarization. In a later study, Brixner et al. $(2004)^{56}$ reported the ionization of potassium dimer molecules, where dipole transitions are favoured by different directions of the exciting laser field. With this example, we understand directly the advantages of having a single laser beam whose polarization varies in time. Polarization pulse shaped lasers are also an efficient method for nano-optical manipulation. Aeschlimann et al. $(2007)^{57}$ have achieved subwavelength dynamic localisation of electromagnetic intensity on the nanometre scale, overcoming the spatial restrictions of conventional optics by using adaptive polarization shaping of femtosecond laser pulses. This technique has also been used for the generation of ultrashort laser pulse pairs, whose time delay between them is controlled with a zeptosecond precision. ${ }^{58}$ Dozens of other further studies based on this principle have been done in the last fifteen years, feeding a rich literature. This technique of pulse shaping allows one to reach a huge variety of different time varying polarized pulses, but it suffers also from some limitations. The number of SOP contained in the resulting pulse depends directly on the number of LCD, as they are independently tuned to obtain the desired ellipticity. This cannot be enough to cover entire parts of a Poincaré sphere. It also requires a complex setup, involving LCDs, all-reflective zero-dispersion compressor, interferometers and computer resources, the desired polarization being computed with special algorithms. Recent advances in polarization state generation (PSG) involve arbitrary polarization synthesis by a plasmonic nanoantenna fed by the coherent control of two input waveguides ${ }^{59}$ and parallel architecture based on digital micromirror devices. ${ }^{60}$

Another class of polarized beams has been theoretically developed later in order to cover the whole Poincaré sphere of polarization. The so-called "Full Poincaré Beams" have been introduced by Beckley et al. $(2010)^{61}$ and consists in a superposition of a Gaussian mode and a spiral-phase Laguerre-Gauss mode having orthogonal polarizations. In this case, and unlike the Brixner pulses, all the polarization states are displayed spatially into the beam.

\subsection{Polariton sweeping on the Poincaré Sphere}

We can now take advantage of a feature that is usually regarded as a shortcoming of microcavity polaritons, but that in our case will turn the simple effect just proposed into a mechanism that powers a new type of light. This effect would thus provide both of the mechanisms we have mentioned before, namely (i) full Poincaré beams, (ii) in time. In the panels of Fig. 5 is highlighted the realization of a contro-polarized two-pulse coherent control experiment. This is achieved through the full exploitation of the setup previously introduced, (see the details of the two excitation arms with polarization control in Fig. 3). The top two rows in Fig. 5 report the time behavior of the cross-section density of the polariton emission resolved in the two spin populations, right (R, blue, first panel) and left (L, red, second panel) circular. The starting time of the two spin emission is correspondent to the arrival of the two pulses, respectively. As it can be seen the two spin populations oscillates mutually independent, 


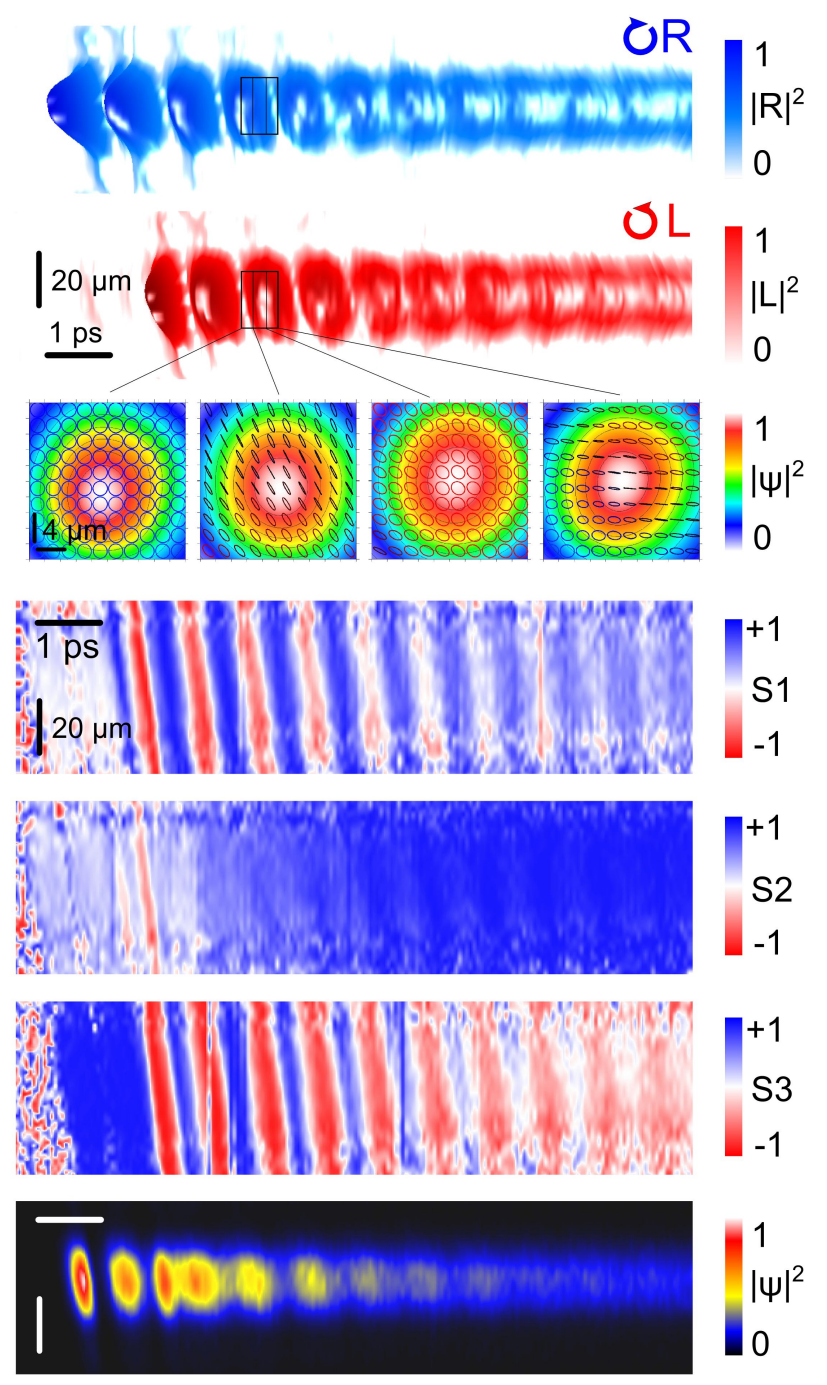

Figure 5. Experimental polarized Rabi oscillations and contro-polarized two pulse coherent control for polarization shaping. The top two panels show the time-space charts of the polariton amplitude for the right $(R$, blue) and left $(L$, red) circular polarization. Their Rabi oscillations remain decoupled. In the third row the spatial distributions of the density and polarization texture at $200 \mathrm{fs}$ time intervals during one of the initial cycles after the second pulse arrival. The emitted intensity scale in each panel has been normalized to its top density but it is remains basically constant (color scale in the bottom row chart), after the second pulse arrival On the other hand, the oscillations are transferred to the polarization texture. The fourth, fifth and sixth panels show the time-space charts for the three Stokes parameter, $S_{1}, S_{2}$ and $S_{3}$. The Rabi oscillations in such panels are clearly seen after the arrival of the second pulse. The last panel shows the overall density. Data readapted from Colas et al. (2015). ${ }^{17}$

nine or ten oscillations can be resolved before the polariton dephasing leads to the oscillations quench. Here we have set a relative time delay to achieve the condition of anti-Rabi phase, $\Phi=1.5 T_{R}$. Such half-integer value is a good condition to transfer the density oscillations in each of the two spin to polarization oscillations. The resulting polarization texture can be observed in the real-space maps of the third row, where the normalized total density profile is overlapped too in a central square area of $20 \times 20 \mu \mathrm{m}^{2}$. The four time snapshots, at almost regular intervals of $200 \mathrm{fs}$, corresponds to the four vertical lines of the small boxes in the upper two rows. We see that right circular, antidiagonal linear, left circular and horizontal linear states are alternating in the ultrafast sequence. The associated Stokes parameter are evolving in time as highlighted by the following three time-space 
panels. Finally the bottom row, presenting the total density through time, shows that the initially high visibility Rabi time fringes are strongly (yet not completely) quenched by the arrival of the second, contropolarized and Rabi-antiphases pulse. The intensity oscillations quench can be improved upon a calibration not only of the time delay, but also of the two pulse relative power ratio.

Now, we have already discussed the significant lifetime imbalance between the two types of polaritons. We know that the upper polariton enjoys a much shorter lifetime than its homologue the lower polariton, regardless of the polarization. From the fits realized on the previous Rabi experiments, we have found the upper polariton lifetime is typically of the order of $2 \mathrm{ps}$ while it is of the order of $10 \mathrm{ps}$ for the lower polariton. However these short values can be now tuned by orders of magnitudes in different samples. As an example, microcavities presenting particles lifetime of the order of the hundred of picoseconds have been demonstrated by M. Steger et al. (2013). ${ }^{62}$ In our case, the lifetime imbalance between the polaritons leads to time-dependent Rabi oscillations converging toward a monotonously decaying signal as the population of the upper polaritons "evaporates" and only lower polaritons remain. The decay of the polariton fields leads to a continuous drift on the Poincaré sphere, starting by describing the initial circle of the Rabi dynamics in the absence of dissipation, toward a final point defined by the polarized lower polaritons. This has, as an interesting consequence, the emission of a light "visiting" a plethora of states of polarization. In Fig. 6 are shown two realization cases spanning different areas of the Poincaré sphere, which is reported as a cylindrical projextion to cartesian coordinates. One can see how the polarization evolves, starting from the north pole line of right circular spin corresponding to the first right polarized pulse, circles and starting to rotate over the sphere after the second, contro-polarized pulse arrival. Each circle is drawn in one Rabi cycle which in the present case is about $800 \mathrm{fs}$. The arrows on the trajectories follow the direction of time toward the final state, which here corresponds to almost linear polarization states of prevalently diagonal ( $D$, on the left, solid blue trajectory) and anti-diagonal $(A$, on the right, dashed red trajectory) degree.

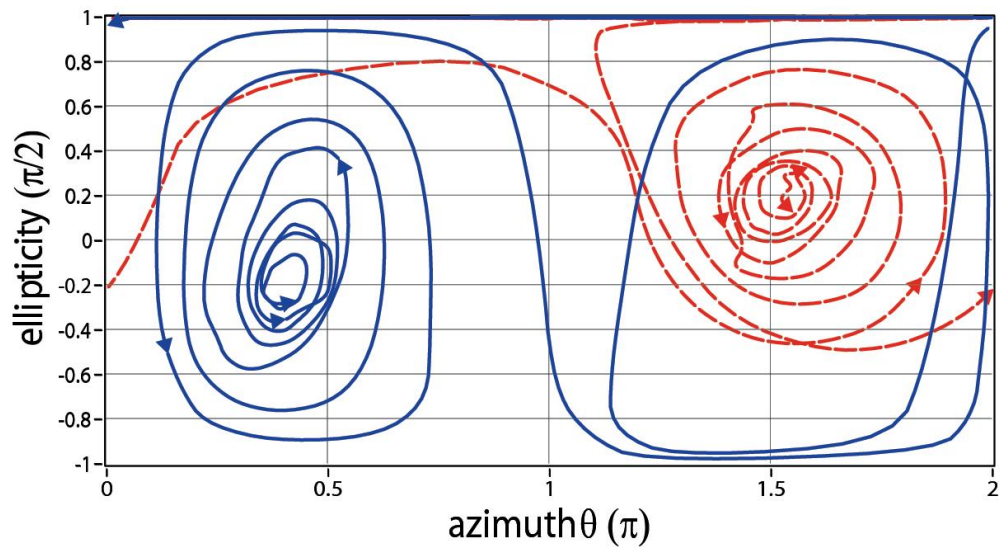

Figure 6. Polarization shaping experiments plotted on the cylindrical projection of the Poincaré sphere. The polarization oscillations are realized upon two contro-circular pulses excitation with half-integer time delay on the scale of the Rabi period: $\Phi=n \cdot 0.5 T_{R}$ with $n$ odd number. The final state has a degree of circular polarization (ellipticity) depending on power balance, while its specific linear direction (azimuth) is set by the optical phase delay $\phi$.

\section{TOPOLOGY TWIST}

Here we show how to resonantly initialize the polariton condensate with non-homogeneous space textures of polarization, and their dynamical modification in the linear regime. In the specific, we realize a full Poincaré pattern such as the skyrmion, whose reshaping on time lengths of the order of the polariton lifetimes can be described as a twist in the polarization space and reveal a wide framework of generalized skyrmions and spin vortex states. ${ }^{18}$ Again, the recent works we show here point to the large potentialities of microcavity polaritons and of their topological resonant excitation (e.g., associated to the presence of orbital angular momentum vortex states with non-trivial polarization textures), whose dynamical redistribution represents a polarization shaping approach fully exploitable in engineered microcavity devices. 
On the one hand, the polarization dynamics in semiconductor microcavities is often ascribed to spin-orbitallike coupling terms, such as the transverse-electric-transverse-magnetic (TE-TM) modes' splitting resulting in the so the called optical spin Hall effect. ${ }^{63-65}$ Such term can exert a precessing action on the pseudospin vector, generating differently polarized regions even when starting from a homogeneous polarization. ${ }^{66,67}$ However in this case, it is usually needed the presence of an imprinted or nonlinearly-activated radial or at least inhomogeneous phase gradient, whose associated in-plane $k$ is hence differently oriented with respect to the initial homogeneous polarization. On the other hand, it is sometimes useful to consider also the presence of a $k$-independent anisotropy term $\chi_{0}$ associated, e.g., to strain effects, ${ }^{68,69}$ that finally results in the dephasing between two given linear polarizations, say $x$ and $y$, and hence in the change of any non-eigenmode that can be expressed in that basis. Such term is responsible for the polarization reshaping observed here.

The experimental realization is carried upon a modification of the previous scheme in Fig. 3. Here we use only one of the two excitation arms on the top-left side (single pulse experiment). However, the single excitation pulse should be thought as sculpted by two different topologies in the two spin, respectively a $L G_{01}$ vortex of unitary winding number and a Gaussian with zero azimuthal number $L G_{00}$. Such configuration can be achieved by proper use of a single $q$-plate, ${ }^{70}$ an anisotropic and inhomogeneous liquid-crystal device which in the last years has unfolded a rich exploration of optical vorticity, with recent applications to full- and half-quantum vortex dynamics in polariton condensates ${ }^{71}$ too. In the present case, the exciting pulse is represented by a laser pulse (4 ps duration and $0.5 \mathrm{~nm}$ bandwidth) which is tuned to selectively excite only the LPB mode. Indeed, we are not exploting the Rabi oscillations as before, but the peculiarity of microcavity polaritons stated above, that consists in the anisotropy splitting of linear polarizations along preferential axis of the samples. By means of polarization control of the incoming/outgoing pulse and tuning of the $q$-plate, we can set a specific superposition of two $L G$ beams with integer or null phase winding in the two spin components: their combination is responsible for the resultant vector vortex beam. ${ }^{72}$ One relevant field pattern that is possible to obtain, among the others, is the skyrmion $\left(\right.$ see $\left.^{18}\right)$, which we imprint on the photonic pulse and hence in the initial resonant polariton state. Upon specific conditions the skyrmion corresponds to the stereographic projection of the whole Poincare sphere itself $\left(\right.$ see also $^{61}$ ), and it should be considered the full-Poincaré beam by excellence.

Our experimental skyrmions and their reshaping have been backed-up by theory and a numerical model, whose results are reported in Fig. 7. As shown on the left side column, projected onto the circular polarization basis, the skyrmions are characterized by the presence of an integer phase winding (orbital angular momentum) in one of the spin components and a zero-winding in the opposite one. The resultant vectorial field exhibits an inhomogeneous pattern comprising all polarization states, as typical of full Poincaré beams (see the first panel of the central column of Fig. 7). The skyrmions possess a specific circumference of full linear polarization ( $l$-line) and they are fingerprinted by the inversion of the sign of circular polarization degree when crossing this circumference in the radial direction. The $l$-line is conformally mapped to the equatorial loop of the Poincaré sphere (right column) while the inner and outer regions to such circle corresponds to the two hemispheres of the sphere, respectively. Indeed the polarization changes from right-circular at the centre to left-circular at large distance (or viceversa). Two fundamental types of textures can be obtained, the star-like or the lemon-like skyrmion, ${ }^{18,73}$ depending on the winding direction along the equatorial line with respect to that in the real-space. In any case, the radial lines in real-space map to the meridians of the sphere, while the circle lines in real-space map to the parallels of the sphere. The conformal mapping of the polarization sphere to the real-space mantains the angles between these lines. We point out that by moving the light point of the stereographic projection into the sphere, the skyrmion $l$-line would gradually move towards infinitity in real-space, obtaining the polarization field of an infinite-size $\mathrm{HQV},{ }^{74}$ associated to only one emisphere.

In order to represent the dynamical behavior of our experimental observations, we used numerical computation implementing a two-component open-dissipative Gross-Pitaevskii model describing the system, including the anisotropy splitting term $\chi_{0}$. The polarization textures reshaping at four successive time frames is reported in the central column, highlighting a complex redistribution comprising spin transport flows across the $l$-line. However, the reshaping is decoupled from both the mass transport, given the linear regime, and from the $\left(\sigma_{+}\right)$ phase singularity movement, which remains in the centre (not shown). The dynamics can be projected onto each of the three Stokes basis for polarization states, as shown in the small panels (three for each time frame) in the third column. At the intermediate time here shown $(\sim 30 \mathrm{ps})$, noteworthy the $S_{2}$ and $S_{3}$ distributions in real 

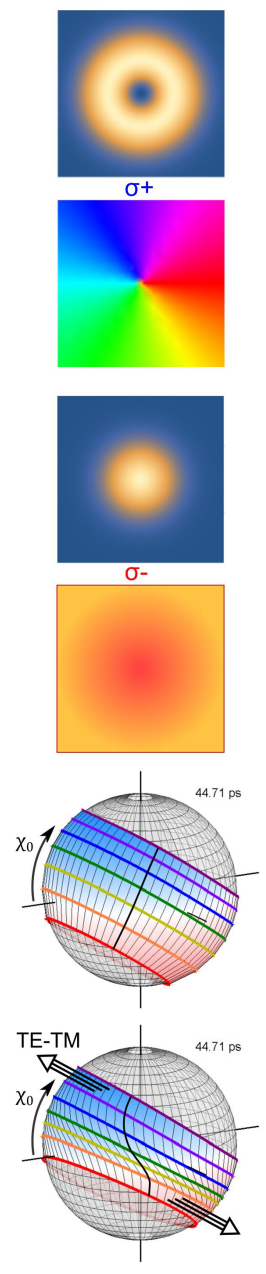

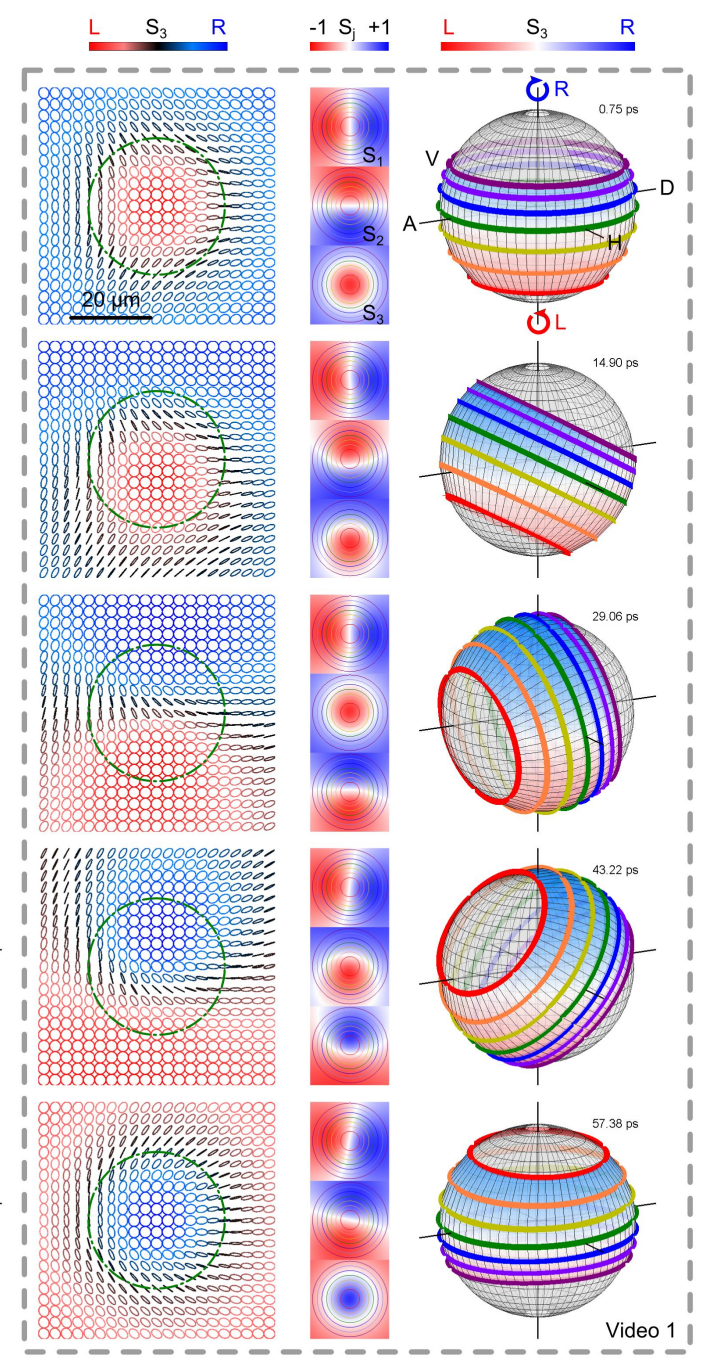

Figure 7. Skyrmion twist, numerical simulation. On the left column top panels are reported the density and phase maps of the initial two spin components $\left(\sigma_{+} \equiv R\right.$ and $\left.\sigma_{-} \equiv L\right)$ of the skyrmion. The central column reports instead the polarization texture at initial and subsequent time snapshots, where the colour scale is proportional to the circular degree of polarization $S_{3}$. The $l$-line of linear polarization is reported as a dashed green circle. The three $S_{1,2,3}$ Stokes degree of polarizations at different times are plotted in the small panels on the right. Several concentric circles in real space (each represented with a different color) are then conformally mapped onto the Poincaré sphere on the right column. The anisotropy splitting $\chi_{0}=0.08 \mathrm{meV}$ between the horizontal $(H)$ and vertical $(V)$ polarizations produces a dynamical twist of the whole texture on the Poincaré sphere, leading at around 30 ps to a $90^{\circ}$ twisted skyrmion where the $S_{2}$ and $S_{3}$ distributions in real space have roughly swapped with respect to initial time. At even longer time $(\sim 60$ ps $)$ the $180^{\circ}$ twist corresponds to a complete transmutation of the initial star skyrmion into its conjugate state (lemon skyrmion). The effect is discussed in Donati et al. (2016). ${ }^{18}$ The two left bottom panels $\left(\chi_{0}=0.02 \mathrm{meV}\right)$ show the additional effect of including the TE-TM splitting, which leads to a transverse shrinking and an azimuthal straining of the topological belt. See also Video 1. http://dx.doi.org/10.1117/12.2250997.1

space have roughly exchanged with each other, with respect to initial time. The clearest picture of the complex reshaping appears however upon plotting on the Poincaré sphere, as shown in the last column. The real-space loops of the skyrmion, which initially map to the parallels on the sphere, rotate during time around the $S_{1}$ basis axis associated with the anisotropy directions in real-space (i.e., $x y \equiv H V$ ). At the latest time here shown $(\sim 60 \mathrm{ps})$ the twist reaches $180^{\circ}$, i.e., to the reversal of the initial star skyrmion into its conjugate state (lemon 
skyrmion). The complete topology-flip has exchanged $R$ and $L$ spin population between the inner and outer regions to the $l$-line. Similar twists are observed when starting with opposite skyrmions or double-loop topologies such as the hedgehog and hyperbolic spin vortices. The twist speed is constant over time, as the effect is linear, independent on the density of polaritons which are leaking out of the microcavity according to an exponential law. The normalized twist speed retrieved from the numerical simulation is about $\beta / \chi_{0} \sim 40^{\circ}(\mathrm{ps} \cdot \mathrm{meV})^{-1}$, as expected also by a theoretical evaluation. Moreover, such velocity is the same for any circle: the real-space circular corona maps to a "topological belt" which is twisting rigidly. Finally, as shown in the bottom left panels of Fig. 7, we point out that the simultaneous presence of a finite TE-TM splitting term, is summing up to the $\chi_{0}$ anisotropy, resulting in a transverse shrinking and azimuthal straining of the topological belt. This effect would be influenced by a large density regime, given the nonlinear push of radial flows associated to finite radial $k$, summing up to the azimuthal flows of the vortex charge. We hope that the visualization of the reshaping dynamics of the full Poincaré topology on the Poincaré sphere itself and the description in terms of generalized skyrmions and spin vortices can help shedding light on a variety of topology states and dynamics and in the perspective of possible spintronics and polarization shaping technologies.

\section{QUANTUM VORTICES}

Following the footsteps of the previous experiments on the coherent control of the polariton Rabi oscillations and the polariton polarization shaping, we introduced and observed the generation of Rabi-powered vortex oscillations in real space. This is achieved by using a series of two pulses, the first one creating a stable vortex in the polariton condensate, and the second one disturbing the density's homogeneity leading to the vortex oscillations. These results can be reproduced by using standard coupled-Schrödinger Equations for the polaritons, fed with the adequate initial state. The different decay sources present in the system can be added in the Hamiltonian in order to model the oscillations damping. The system can also be solved formally and analytical solutions can be derived. To get more insights into this dynamics, we study the evolution of the orbital angular momentum (OAM) and see its connection with the Rabi coupling.

Unlike their classical counterparts, quantum vortices appear to be stable configurations in a superfluid. These "topological charges", characterized by a quantized circulation $\oint \vec{v} \cdot \overrightarrow{d l}=\frac{2 \pi h}{m}$ have been initially predicted by Onsager in 1949 in his work on superfluid Helium. But these predictions were only widely accepted after Feynmans contributions in 1955. Further theoretical developments have also been made by Gross and Pitaevskii (1961). Quantum vortices have been then observed in a wide range of systems generally described by a complex wave function whose phase plays a key role in their dynamics. We can mention superconductors ${ }^{75}$ where the vortex dynamics is mainly governed by the thermal and quantum fluctuations. Such vortices can be as well manipulated by applying external forces, such as electric currents. Superconductor vortices were extensively studied as they can carry Abrikosov vortices (fluxons) and exhibit some complex ordering like Abrikosov lattices, spin glasses etc. Bose-Einstein Condensates (BEC) are also a good platform to generate quantum vortices since they are superfluid systems. ${ }^{76}$ The vortex generation in a BEC has been first demonstrated by Cornell's group in $1999^{77}$ following his previous works on BEC for which he was awarded the Nobel prize in 2001. It was later demonstrated that vortices can even appear spontaneously during phase transitions in which the BEC formation occurs, ${ }^{78}$ and the reconnection of vortex lines has been studied in the framework of superfluid turbulence. ${ }^{79}$ Vortex lattices were also observed in rotating BEC by the group of W. Ketterle, ${ }^{80}$ exhibiting a high vortex lifetime $(\approx 1 \mathrm{~min})$. Another interesting feature of the vortices produced in atomic BECs and related to our polariton vortices behaviours, is their core's precession. The phenomenon was characterized and observed by B. P. Anderson et al. $(2000)^{81}$ in a BEC made of a superposition of two internal states of ${ }^{87} \mathrm{Rb}$. The vortex motion was in this case attributed to a Magnus effect applied on the vortex into the quantum fluid. More recently, it has been demonstrated that similarly to optical fields,${ }^{82-84}$ electron beams could also propagate with a phase singularity, ${ }^{85}$ giving a new degree of freedom to the electrons. Shortly later, electron vortex beams with high quanta of angular momentum (up to $100 \hbar$ ) were realized ${ }^{86,87}$ leading to potential applications in electron microscopy of magnetic and biological specimens.

The polariton community was of course not left behind in the investigation of vortex experiments. Lagoudakis et al. $(2008)^{12}$ have reported the formation of pinned single quantized vortices in the Bose-condensed phase of a polariton fluid, giving new clues to understand the superfluid nature of the polariton condensates. Quantum 
half-vortices were then observed in polariton condensates ${ }^{88}$ by the same group, following their prediction by Yu. Rubo. ${ }^{89}$ The full-vortex twin-charge splitting ${ }^{90}$ dynamics pinned by defects was later observed. We can also mention the observation of metastable persistent polariton superflows, ${ }^{10}$ where it was shown how sustained quantized angular momenta can be transferred to the steady-state condensate, insuring a long-lived vorticity. The effect of the nonlinearity on the vortex size was discussed by Krizhanovskii et al. ${ }^{91}$ and by Voronova et al. (2012). ${ }^{92}$ More elaborated topological structures were introduced, like the formation of a vortex chain, achieved in a resonantly pumped polariton superfluid. ${ }^{93} \mathrm{~A}$ circular chain containing 8 vortices of the same charge was generated with a Laguerre-Gauss beam, paving the way for more experiments involving self-arranged and same-sign vortex lattices. Other recent advances on the field include the generation of unconventional states, such as polarization textures corresponding to spin vortices in open-cavity polaritons, ${ }^{94}$ or exotic topologies (e.g., those referred to as generalized skyrmions in the previous section) under confined nonresonant ${ }^{95}$ and imprinted resonant ${ }^{18}$ excitation dynamics. Intriguing $2 \mathrm{D}+\mathrm{t}$ topological strings have been associated to the half-vortex precession, together with pair generation phenomena at high density. ${ }^{71}$ In the considered polariton system, the vortex dynamics is ruled by the interplay between the nonlinearity and the disorder landscape.

In the following, we will deal with polariton vortices in the linear regime, where the vortices are directly imprinted to the condensate through the laser excitation. Thus, the size of the vortices in the different components will not be affected by any nonlinearity as it the case for interacting condensates. In order to analyze the vortex dynamics, will also focus on the OAM value for the different polariton components. Several applications are based on the OAM properties. For example, quantum correlations between the OAM variables can be used in quantum information ${ }^{96}$ processing and notably in protocols for quantum key distribution. ${ }^{97}$ The OAM based multiplexing was developed and used to increase the efficiency of millimetre-wave wireless communications ${ }^{98}$ and to confer robustness to free-space communications, ${ }^{99}$ with demonstration of high accuracy transmission over distances of more than $100 \mathrm{~km} .{ }^{100}$ Compact plasmonic metasurfaces can be designed for the spin-orbit coupling conversion of the spin into an arbitrary OAM photonic vortex,${ }^{101}$ while new classes of lasers have been proposed to generate all the states of an higher-order Poincaré sphere. ${ }^{102}$ The data exchanged between OAM beams was reported to reaches Terabit rates values in free-space transmission ${ }^{103}$ and fibers, ${ }^{104}$ and recently a scheme for the time-division multiplexing of photonic pulses carrying different orbital angular momentum has been introduced. ${ }^{105}$ Topological light was also proposed to study new selection rules and manipulation of the ionized state in photoionization processes ${ }^{106}$ thus enriching the scenarios of femtochemistry. Proposals of polariton vortices for information processing ${ }^{107}$ have been made as well as a scheme for gyroscopes ${ }^{108}$ that exploits the macroscopic response of the system to small perturbations to create sensitive devices.

\subsection{Swirling Rabi vortices}

We have already said that vortex oscillations can be obtained by sending a Gaussian pulse on an unperturbed vortex, the vortex core oscillating circularly into the beam. We have experimentally observed polariton vortex oscillations that are rapidly damped after a few picoseconds, the vortex becoming immobile again. As the vortex oscillations are powered by the Rabi oscillations between the photonic and excitonic field, we suspect that the lifetime imbalance between the upper and lower polaritons plays as well a key role in this mechanism.

The system can be simply modeled by two coupled-Schrödinger Equations to which we add the excitation scheme, i.e. a Laguerre-Gauss pulse (carrying the vortex phase singularity) followed by a Gaussian pulse. One can see in Fig. 8(a) the basic vortex dynamics obtained from the computational model. Here, the Laguerre-Gauss pulse is sent at early time to generate the vortex, and the Gaussian pulse is sent at $t_{02} \approx 4 \mathrm{ps}$. After the first pulse, the vortex is perfectly centered into the beam and motionless. After the second pulse, the minimum of intensity no longer remains in the center of the beam, as shown on the density plot, which induces the vortex oscillations. The dynamical position of the vortex is displayed with a $3 \mathrm{D}$ curve for both photonic (in red) and excitonic fields (in blue). The vortices trajectory in space and time looks helical but it is actually not, the angular velocity varying periodically in time. The two vortices, in the photon and in the exciton, rotates around each other at the Rabi frequency similarly to a Newton's cradle, one vortex slowing down while the other accelerates. In the case of Fig. 8(b) the upper polariton lifetime was included in the Hamiltonian. The vortex precession now follows a spiral whose radius damping corresponds to the UP lifetime, here 4ps. The vortex in both photonic and excitonic field converges towards the same fixed point. To understand these vortex motions, it is interesting to look at the vortex behaviours through the eigenstates of the system, namely by looking at the polaritonic fields 
(a)

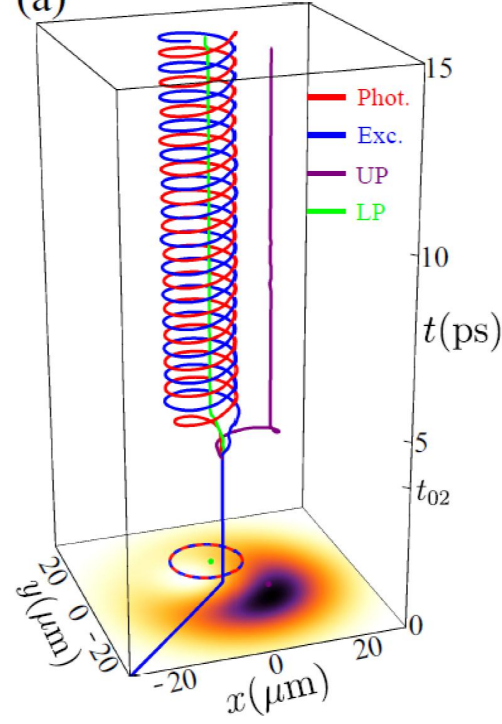

(b)

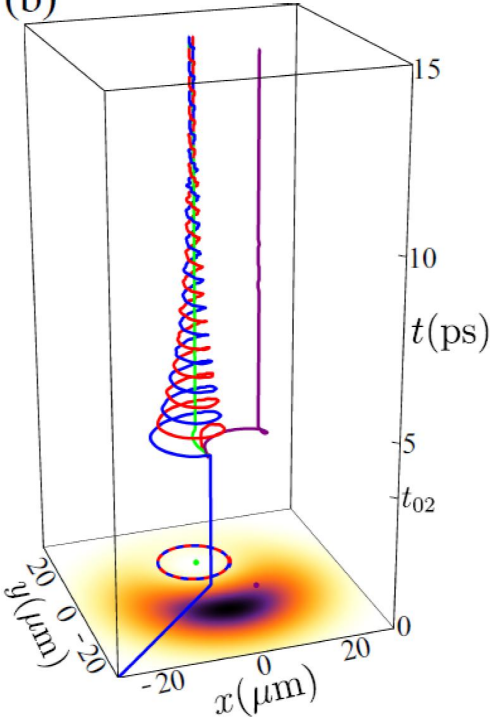

Figure 8. Vortex oscillations generated by perturbing the system, that was initially prepared in a vortex state, with a Gaussian pulse sent at $t=t_{02} \approx 4 \mathrm{ps}$. The vortex trajectory in the photonic (excitonic) field is plotted in red (blue) and also in the upper (lower) field in purple (green). The wave function intensity $\left|\psi_{C}\right|$ is plotted in the $x y$ plane for a $t>t_{02}$, where are as well projected the vortex trajectories. (a) Conservative case, without including decay. After the second pulse, the vortices in both fields describe circles with a non constant angular velocity. (b) Adding the upper polariton decay, it results in a damping of the oscillations at the rate of the UP decay.

$\psi_{+}$and $\psi_{-}$which are obtained by combining the bare states. The vortex trajectories in the polaritonic fields are plotted with green (lower) and purple (upper) lines in Fig. 8. Before the Gaussian pulse, the vortex is located in the center of the beam for all the fields. After the second pulse, we see that unlike in the photon and exciton fields, into which the vortex oscillates, it is simply shifted to the periphery of the beam in the polaritonic fields, without exhibiting any oscillations. In the damped case, we see now clearly that as the upper field is dying, the vortex in the photon/exciton field converges toward the position of the lower one. We know now that the oscillations occur because the vortex of the polaritonic fields are shifted at different position into the beam, the vortex in the photon/exciton components oscillating between them. Yet the vortex oscillations are located close to the vortex position in the LP, possibly due to the mass imbalance between the two fields.

More insight of the vortex dynamics can be obtained by looking at the value of the OAM for the different fields. For the polaritons, the OAM is conserved through the sum of the OAM of each sub-fields (photon/exciton), which allows the OAM of the individual fields to vary. In any case $d\left(\langle\widehat{L}\rangle_{\psi_{C}}+\langle\widehat{L}\rangle_{\psi_{X}}\right) / d t$ has to be 0 . The total number of particles $N_{\psi}$ is similarly conserved through the sum of the different components $N_{\psi_{\mathrm{C}}}+N_{\psi_{\mathrm{X}}}$. However, it is not the case for the OAM per particle defined as the ratio $l_{\psi}=\langle L\rangle_{\psi} / N_{\psi}$. In order to illustrate these conservation rules, we have computed the different OAM for the conservative case of the oscillating vortex created with two pulses (see Fig. 8(a)). One can see in Fig. 9(a) the time evolution of the fields intensities $N_{\psi}$. The bare fields $\psi_{C}$ (in red) and $\psi_{X}$ (in blue) exhibit Rabi oscillations while the polaritonic fields $\psi_{+}$(in purple) and $\psi_{-}$ (in green) do not. The different intensities of course increase after the second pulse arrival, the system being filled with more particles. The conserved quantities are plotted in orange. The OAM is plotted in (b) for the different fields. One can see that the OAM of the bare fields oscillates exactly between the OAMs steady value of the polaritonic fields. The OAM for the different fields is not be affected by the second pulse, that initiates the vortex oscillations process. Which makes sense considering that the Gaussian does not carry additional angular momentum. Nevertheless, the vortex oscillations appear more explicitly when one computes the OAM per particle for the different fields, as plotted in (c). When the vortices are exactly in the center of the beam, that is before the second pulse, the angular momentum per particle is exactly quantized to one unit of $\hbar$. After 

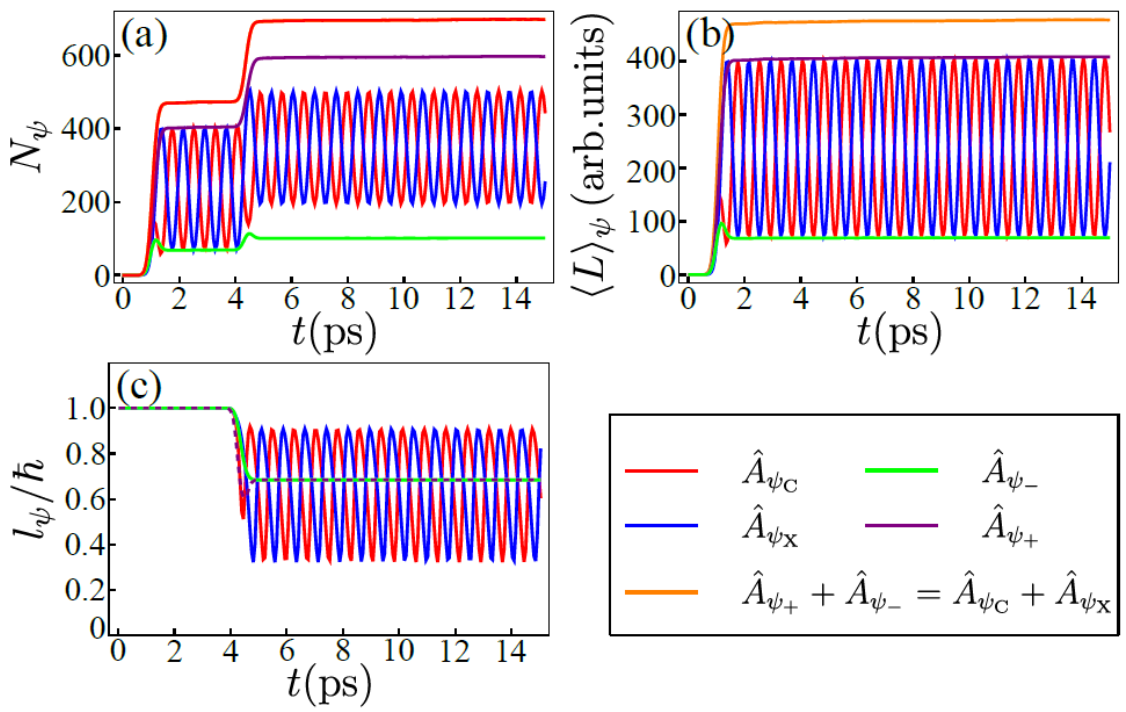

Figure 9. Numerical dynamics of a topological two-pulse excitation. Various OAM computed from the two-pulse experiment simulation data, corresponding to the case of a first Gaussian pulse and a second Vortex pulse. (a) Fields intensities. (b) Angular Momentum for the different fields. (c) Angular momentum per particle, normalized in units of $\hbar$. See the legend on the right bottom corner for the color code of the operators $\widehat{A}(\langle L\rangle, N$ or $l)$ applied to the corresponding field. The conserved quantity are plotted in orange.

the second pulse, the vortices in the polariton fields do not oscillate but are displaced to a fixed position on the periphery of the beam (see purple and green lines in Fig. 8), with a corresponding value of $l$ which is lower than 1. As mentioned by Pitaevskii and Stringari, ${ }^{109}$ for a vortex core displaced from the center of the beam, the OAM per particle takes a value lower than 1 , the axial symmetry of the problem being lost. Here, the polaritonic vortices are symmetrically displaced into the beam, $l_{\psi_{+}}$and $l_{\psi_{-}}$thus share the same value. In the photonic and excitonic fields, the vortices oscillate from the center to the periphery in circle, so that the variation range of the OAM per particle oscillations is constant. Since the vortex motion is directly powered by the Rabi oscillations, we expect that the accurate control of these new topological strings could be achieved in the same way that we performed the control of the quantum state or the polarization state in our previous experiments.

\section{ACKNOWLEDGMENTS}

We acknowledge Romuald Houdré for the growth of the microcavity polariton device and Alberto Bramati for the related know-how, Lorenzo Marrucci and Bruno Piccirillo for the $q$-plate devices and the related know-how. We acknowledge the project ERC POLAFLOW (Grant 308136) for financial support.

\section{REFERENCES}

[1] Byrnes, T., Kim, N. Y., and Yamamoto, Y., "Exciton-polariton condensates," Nat. Phys. 10(11), 803-813 (2014).

[2] Kasprzak, J., Richard, M., Kundermann, S., Baas, A., Jeambrun, P., Keeling, J. M. J., Marchetti, F. M., Szymańska, M. H., André, R., Staehli, J. L., Savona, V., Littlewood, P. B., Deveaud, B., and Dang, L. S., "Bose-Einstein condensation of exciton polaritons.," Nature 443(7110), 409-414 (2006).

[3] Balili, R., Hartwell, V., Snoke, D., Pfeiffer, L., and West, K., "Bose-Einstein condensation of microcavity polaritons in a trap," Science 316(5827), 1007-1010 (2007).

[4] Whittaker, C. E., Dzurnak, B., Egorov, O. A., Buonaiuto, G., Walker, P. M., Cancellieri, E., Whittaker, D. M., Clarke, E., Gavrilov, S. S., Skolnick, M. S., and Krizhanovskii, D. N., "Polariton pattern formation and its statistical properties in a semiconductor microcavity," arXiv:1612.03048 [cond-mat] (2016). arXiv: 1612.03048 . 
[5] Dominici, L., Petrov, M., Matuszewski, M., Ballarini, D., De Giorgi, M., Colas, D., Cancellieri, E., Silva Fernández, B., Bramati, A., Gigli, G., Kavokin, A., Laussy, F., and Sanvitto, D., "Real-space collapse of a polariton condensate," Nat. Commun. 6, 8993 (2015).

[6] Dreismann, A., Cristofolini, P., Balili, R., Christmann, G., Pinsker, F., Berloff, N. G., Hatzopoulos, Z., Savvidis, P. G., and Baumberg, J. J., "Coupled counterrotating polariton condensates in optically defined annular potentials," Proc. Natl. Acad. Sci. 111(24), 8770-8775 (2014).

[7] Roumpos, G., Lohse, M., Nitsche, W. H., Keeling, J., Szymańska, M. H., Littlewood, P. B., Löfller, A., Höfling, S., Worschech, L., Forchel, A., and Yamamoto, Y., "Power-law decay of the spatial correlation function in exciton-polariton condensates," Proc. Natl. Acad. Sci. 109(17), 6467-6472 (2012).

[8] Amo, A., Pigeon, S., Sanvitto, D., Sala, V. G., Hivet, R., Carusotto, I., Pisanello, F., Leménager, G., Houdré, R., Giacobino, E., Ciuti, C., and Bramati, A., "Polariton superfluids reveal quantum hydrodynamic solitons.," Science 332(6034), 1167-1170 (2011).

[9] Pigeon, S., Carusotto, I., and Ciuti, C., "Hydrodynamic nucleation of vortices and solitons in a resonantly excited polariton superfluid," Phys. Rev. B 83(14), 144513 (2011).

[10] Sanvitto, D., Marchetti, F. M., Szymańska, M. H., Tosi, G., Baudisch, M., Laussy, F. P., Krizhanovskii, D. N., Skolnick, M. S., Marrucci, L., Lemaître, A., Bloch, J., Tejedor, C., and Viña, L., "Persistent currents and quantized vortices in a polariton superfluid," Nat. Phys. 6(7), 527-533 (2010).

[11] Amo, A., Lefrère, J., Pigeon, S., Adrados, C., Ciuti, C., Carusotto, I., Houdré, R., Giacobino, E., and Bramati, A., "Superfluidity of polaritons in semiconductor microcavities," Nat. Phys. 5(11), 805-810 (2009).

[12] Lagoudakis, K. G., Wouters, M., Richard, M., Baas, A., Carusotto, I., André, R., Dang, L. S., and Deveaud-Plédran, B., "Quantized vortices in an exciton-polariton condensate," Nat. Phys. 4(9), 706-710 (2008).

[13] Manni, F., Lagoudakis, K. G., Paraïso, T. K., Cerna, R., Léger, Y., Liew, T. C. H., Shelykh, I. A., Kavokin, A. V., Morier-Genoud, F., and Deveaud-Plédran, B., "Spin-to-orbital angular momentum conversion in semiconductor microcavities," Phys. Rev. B 83, 241307 (2011).

[14] Toledo-Solano, M., Mora-Ramos, M. E., Figueroa, A., and Rubo, Y. G., "Warping and interactions of vortices in exciton-polariton condensates," Phys. Rev. B 89, 035308 (2014).

[15] Flayac, H., Shelykh, I. A., Solnyshkov, D. D., and Malpuech, G., "Topological stability of the half-vortices in spinor exciton-polariton condensates," Phys. Rev. B 81, 045318 (2010).

[16] Dominici, L., Colas, D., Donati, S., Restrepo Cuartas, J. P., De Giorgi, M., Ballarini, D., Guirales, G., López Carreńo, J. C., Bramati, A., Gigli, G., del Valle, E., Laussy, F. P., and Sanvitto, D., "Ultrafast control and Rabi oscillations of polaritons," Phys. Rev. Lett. 113, 226401 (2014).

[17] Colas, D., Dominici, L., Donati, S., Pervishko, A. A., Liew, T. C., Shelykh, I. A., Ballarini, D., De Giorgi, M., Bramati, A., Gigli, G., Valle, E. d., Laussy, F. P., Kavokin, A. V., and Sanvitto, D., "Polarization shaping of Poincaré beams by polariton oscillations," Light Sci. Appl. 4(11), e350 (2015).

[18] Donati, S., Dominici, L., Dagvadorj, G., Ballarini, D., De Giorgi, M., Bramati, A., Gigli, G., Rubo, Y. G., Szymańska, M. H., and Sanvitto, D., "Twist of generalized skyrmions and spin vortices in a polariton superfluid," Proc. Natl. Acad. Sci. 113(52), 14926-14931 (2016).

[19] Schumacher, B., "Quantum coding," Phys. Rev. A 51, 2738 (1995).

[20] Nielsen, M. A. and Chuang, I. L., [Quantum computation and quantum information], Cambridge University Press (2000).

[21] Spreeuw, R. and Woerdman, J., "Optical atoms," Progress in Optics 31, 263 (1993).

[22] Faust, T., Rieger, J., Seitner, M. J., Kotthaus, J. P., and Weig, E. M., "Coherent control of a classical nanomechanical two-level system," Nat. Phys. 9, 485 (2013).

[23] Zhu, Y., Gauthier, D. J., Morin, S. E., Wu, Q., Carmichael, H. J., and Mossberg, T. W., "Vacuum Rabi splitting as a feature of linear-dispersion theory: Analysis and experimental observations," Phys. Rev. Lett. 64, 2499 (1990).

[24] Khitrova, G., Gibbs, H. M., Kira, M., Koch, S. W., and Scherer, A., "Vacuum Rabi splitting in semiconductors," Nat. Phys. 2, 81 (2006). 
[25] Matthews, M. R., Anderson, B. P., Haljan, P. C., Hall, D. S., Holland, M. J., Williams, J. E., Wieman, C. E., and Cornell, E. A., "Watching a superfluid untwist itself: Recurrence of Rabi oscillations in a Bose-Einstein condensate," Phys. Rev. Lett. 83, 3358 (1999).

[26] Vasa, P., Wang, W., Pomraenke, R., Lammers, M., Maiuri, M., Manzoni, C., Cerullo, G., and Lienau, C., "Real-time observation of ultrafast Rabi oscillations between excitons and plasmons in metal nanostructures with J-aggregates," Nat. Photon. 7, 128 (2013).

[27] Spreeuw, R. J. C., "A classical analogy of entanglement," Found. Phys. 28, 361 (1998).

[28] Dragoman, D. and Dragoman, M., [Quantum-Classical Analogies], The Frontiers Collection, Springer (2004).

[29] Spreeuw, R. J. C., van Druten, N. J., Beijersbergen, M. W., Eliel, E. R., and Woerdman, J. P., "Classical realization of a strongly driven two-level system," Phys. Rev. Lett. 65, 2642 (1990).

[30] Okamoto, H., Gourgout, A., Chang, C.-Y., Onomitsu, K., Mahboob, I., Chang, E. Y., and Yamaguchi, H., "Coherent phonon manipulation in coupled mechanical resonators," Nat. Phys. 9, 480 (2013).

[31] Kavokin, A., Baumberg, J. J., Malpuech, G., and Laussy, F. P., [Microcavities], Oxford University Press, 2 ed. (2011).

[32] Hennessy, K., Badolato, A., Winger, M., Gerace, D., Atature, M., Gulde, S., Fălt, S., Hu, E. L., and İmamoḡlu, A., "Quantum nature of a strongly coupled single quantum dot-cavity system," Nature 445, 896 (2007).

[33] Weisbuch, C., Nishioka, M., Ishikawa, A., and Arakawa, Y., "Observation of the coupled exciton-photon mode splitting in a semiconductor quantum microcavity," Phys. Rev. Lett. 69, 3314 (1992).

[34] Carusotto, I. and Ciuti, C., "Quantum fluids of light," Rev. Mod. Phys. 85, 299 (2013).

[35] Kasprzak, J., Richard, M., Kundermann, S., Baas, A., Jeambrun, P., Keeling, J. M. J., Marchetti, F. M., Szymanska, M. H., André, R., Staehli, J. L., Savona, V., Littlewood, P. B., Deveaud, B., and Le Si Dang, "Bose-Einstein condensation of exciton polaritons," Nature 443, 409 (2006).

[36] Amo, A., Sanvitto, D., Laussy, F. P., Ballarini, D., del Valle, E., Martin, M. D., Lemaître, A., Bloch, J., Krizhanovskii, D. N., Skolnick, M. S., Tejedor, C., and Viña, L., "Collective fluid dynamics of a polariton condensate in a semiconductor microcavity," Nature 457, 291 (2009).

[37] Liew, T. C. H., Kavokin, A. V., and Shelykh, I. A., "Optical circuits based on polariton neurons in semiconductor microcavities," Phys. Rev. Lett. 101, 016402 (2008).

[38] Amo, A., Liew, T. C. H., Adrados, C., Houdré, R., Giacobino, E., Kavokin, A. V., and Bramati, A., "Exciton-polariton spin switches," Nat. Photon. 4, 361 (2010).

[39] Baumberg, J. J., Kavokin, A. V., Christopoulos, S., Grundy, A. J. D., Butté, R., Christmann, G., Solnyshkov, D. D., Malpuech, G., von Högersthal, G. B. H., Feltin, E., Carlin, J.-F., and Grandjean, N., "Spontaneous polarization buildup in a room-temperature polariton laser," Phys. Rev. Lett. 101, 136409 (2008).

[40] Schneider, C., Rahimi-Iman, A., Kim, N. Y., Fischer, J., Savenko, I. G., Amthor, M., Lermer, M., Wolf, A., Worschech, L., Kulakovskii, V. D., Shelykh, I. A., Kamp, M., Reitzenstein, S., Forchel, A., Yamamoto, Y., and Höfling, S., "An electrically pumped polariton laser," Nature 497, 348 (2013).

[41] Ballarini, D., De Giorgi, M., Cancellieri, E., Houdré, R., Giacobino, E., Cingolani, R., Bramati, A., Gigli, G., and Sanvitto, D., "All-optical polariton transistor.," Nat. Commun. 4, 1778 (2013).

[42] Norris, T., Rhee, J.-K., Sung, C.-Y., Arakawa, Y., Nishioka, M., and Weisbuch, C., "Time-resolved vacuum Rabi oscillations in a semiconductor quantum microcavity," Phys. Rev. B 50, 14663 (1994).

[43] Savona, V. and Weisbuch, C., "Theory of time-resolved light emission from polaritons in a semiconductor microcavity under resonant excitation," Phys. Rev. B 54, 10835 (1996).

[44] Wang, H., Shah, J., Damen, T. C., Jan, W. Y., Cunningham, J. E., Hong, M., and Mannaerts, J. P., "Coherent oscillations in semiconductor microcavities," Phys. Rev. B 51, 14713 (1995).

[45] Marie, X., Renucci, P., Dubourg, S., Amand, T., Jeune, P. L., Barrau, J., Bloch, J., and Planel, R., "Coherent control of exciton polaritons in a semiconductor microcavity," Phys. Rev. B 59, 2494(R) (1999).

[46] Huynh, A., Tignon, J., Roussignol, P., Delalande, C., André, R., Romestain, R., and D. Le Si Dang, "Coherent dynamics of microcavity polaritons in the nonlinear regime," Physica E 13, 427 (2002). 
[47] Brunetti, A., Vladimirova, M., Scalbert, D., Nawrocki, M., Kavokin, A. V., Shelykh, I. A., and Bloch, J., "Observation of spin beats at the Rabi frequency in microcavities," Phys. Rev. B 74, 241101(R) (2006).

[48] Ridolfo, A., Vilardi, R., Di Stefano, O., Portolan, S., and Savasta, S., "All Optical Switch of Vacuum Rabi Oscillations: The Ultrafast Quantum Eraser," Phys. Rev. Lett. 106, 013601 (2011).

[49] Boulier, T., Bamba, M., Amo, A., Adrados, C., Lemaître, A., Galopin, E., Sagnes, I., Bloch, J., Ciuti, C., Giacobino, E., and Bramati, A., "Polariton-generated intensity squeezing in semiconductor micropillars," Nat. Commun. 5 (2014).

[50] Cuevas, A., Silva, B., Carreño, J. C. L., De Giorgi, M., Muñoz, C. S., Fieramosca, A., Forero, D. G. S., Cardano, F., Marrucci, L., Tasco, V., Biasiol, G., del Valle, E., Dominici, L., Ballarini, D., Gigli, G., Mataloni, P., Laussy, F. P., Sciarrino, F., and Sanvitto, D., "Entangling a polariton with one photon: effect of interactions at the single-particle level," arXiv:1609.01244 [cond-mat, physics:physics, physics:quant-ph] (2016). arXiv: 1609.01244.

[51] Nardin, G., Grosso, G., Léger, Y., Piętka, B., Morier-Genoud, F., and Deveaud-Plédran, B., "Hydrodynamic nucleation of quantized vortex pairs in a polariton quantum fluid," Nat. Phys. 7, 635-641 (2011).

[52] Antón, C., Tosi, G., Martín, M. D., Viña, L., Lemaître, A., and Bloch, J., "Role of supercurrents on vortices formation in polariton condensates," Opt. Express 20(15), 16366 (2012).

[53] Schnars, U. and Jüptner, W., [Digital Holography], Springer Berlin Heidelberg (2005).

[54] Brixner, T. and Gerber, G., "Femtosecond polarization pulse shaping," Opt. Lett. 26(8), 557 (2001).

[55] Zhuang, Z., Suh, S.-W., and Patel, J. S., "Polarization controller using nematic liquid crystals," Opt. Lett. 24(10), 694 (1999).

[56] Brixner, T., Krampert, G., Pfeifer, T., Selle, R., Gerber, G., Wollenhaupt, M., Graefe, O., Horn, C., Liese, D., and Baumert, T., "Quantum Control by Ultrafast Polarization Shaping," Phys. Rev. Lett. 92(20) (2004).

[57] Aeschlimann, M., Bauer, M., Bayer, D., Brixner, T., García de Abajo, F. J., Pfeiffer, W., Rohmer, M., Spindler, C., and Steeb, F., "Adaptive subwavelength control of nano-optical fields," Nature 446(7133), 301-304 (2007).

[58] Köhler, J., Wollenhaupt, M., Bayer, T., Sarpe, C., and Baumert, T., "Zeptosecond precision pulse shaping," Opt. Express 19(12), 11638 (2011).

[59] Rodríguez-Fortuño, F. J., Puerto, D., Griol, A., Bellieres, L., Martí, J., and Martínez, A., "Universal method for the synthesis of arbitrary polarization states radiated by a nanoantenna: Synthezising arbitrary polarization states with a single nanoantenna," Laser Photon. Rev. 8, L27-L31 (May 2014).

[60] She, A. and Capasso, F., "Parallel Polarization State Generation," Sci. Rep. 6, 26019 (May 2016).

[61] Beckley, A. M., Brown, T. G., and Alonso, M. A., "Full Poincaré beams," Opt. Express 18(10), 10777 (2010).

[62] Steger, M., Liu, G., Nelsen, B., Gautham, C., Snoke, D. W., Balili, R., Pfeiffer, L., and West, K., "Longrange ballistic motion and coherent flow of long-lifetime polaritons," Phys. Rev. B 88, 235314 (2013).

[63] Kavokin, A., Malpuech, G., and Glazov, M., "Optical spin Hall effect," Phys. Rev. Lett. 95, 136601 (Sep 2005).

[64] Leyder, C., Romanelli, M., Karr, J. P., Giacobino, E., Liew, T. C. H., Glazov, M. M., Kavokin, A. V., Malpuech, G., and Bramati, A., "Observation of the optical spin Hall effect," Nat. Phys. 3, 628-631 (Sept. 2007).

[65] Kammann, E., Liew, T. C. H., Ohadi, H., Cilibrizzi, P., Tsotsis, P., Hatzopoulos, Z., Savvidis, P. G., Kavokin, A. V., and Lagoudakis, P. G., "Nonlinear optical spin Hall effect and long-range spin transport in polariton lasers," Phys. Rev. Lett. 109, 036404 (Jul 2012).

[66] Cilibrizzi, P., Sigurdsson, H., Liew, T. C. H., Ohadi, H., Askitopoulos, A., Brodbeck, S., Schneider, C., Shelykh, I. A., Höfling, S., Ruostekoski, J., and Lagoudakis, P., "Half-skyrmion spin textures in polariton microcavities," Phys. Rev. B 94, 045315 (Jul 2016).

[67] Cilibrizzi, P., Sigurdsson, H., Liew, T. C. H., Ohadi, H., Wilkinson, S., Askitopoulos, A., Shelykh, I. A., and Lagoudakis, P. G., "Polariton spin whirls," Phys. Rev. B 92, 155308 (Oct 2015).

[68] Balili, R., Nelsen, B., Snoke, D. W., Reid, R. H., Pfeiffer, L., and West, K., "Huge splitting of polariton states in microcavities under stress," Phys. Rev. B $\mathbf{8 1}$ (Mar. 2010). 
[69] Klopotowski, L., Martín, M., Amo, A., Viña, L., Shelykh, I., Glazov, M., Malpuech, G., Kavokin, A., and André, R., "Optical anisotropy and pinning of the linear polarization of light in semiconductor microcavities," Solid State Commun. 139, 511-515 (Sept. 2006).

[70] Cardano, F., Karimi, E., Slussarenko, S., Marrucci, L., de Lisio, C., and Santamato, E., "Polarization pattern of vector vortex beams generated by q-plates with different topological charges," Appl. Opt. 51, C1-C6 (Apr 2012).

[71] Dominici, L., Dagvadorj, G., Fellows, J. M., Ballarini, D., De Giorgi, M., Marchetti, F. M., Piccirillo, B., Marrucci, L., Bramati, A., Gigli, G., Szymańska, M. H., and Sanvitto, D., "Vortex and half-vortex dynamics in a nonlinear spinor quantum fluid," Sci. Adv. 1(11), e1500807 (2015).

[72] Schulz, S. A., Machula, T., Karimi, E., and Boyd, R. W., "Integrated multi vector vortex beam generator," Opt. Express 21(13), 16130 (2013).

[73] Bouchard, F., Larocque, H., Yao, A. M., Travis, C., De Leon, I., Rubano, A., Karimi, E., Oppo, G.-L., and Boyd, R. W., "Polarization Shaping for Control of Nonlinear Propagation," Phys. Rev. Lett. 117(23) (2016).

[74] Rubo, Y. G., "Half vortices in exciton polariton condensates," Phys. Rev. Lett. 99, 106401 (Sept. 2007).

[75] Blatter, G., Feigel'man, M. V., Geshkenbein, V. B., Larkin, A. I., and Vinokur, V. M., "Vortices in high-temperature superconductors," Rev. Mod. Phys. 66(4), 1125-1388 (1994).

[76] Leggett, A. J., "Superfluidity," Rev. Mod. Phys. 71(2), S318-S323 (1999).

[77] Matthews, M. R., Anderson, B. P., Haljan, P. C., Hall, D. S., Wieman, C. E., and Cornell, E. A., "Vortices in a Bose-Einstein condensate," Phys. Rev. Lett. 83, 2498 (1999).

[78] Weiler, C. N., Neely, T. W., Scherer, D. R., Bradley, A. S., Davis, M. J., and Anderson, B. P., "Spontaneous vortices in the formation of Bose-Einstein condensates," Nature 455(7215), 948-951 (2008).

[79] Bewley, G. P., Paoletti, M. S., Sreenivasan, K. R., and Lathrop, D. P., "Characterization of reconnecting vortices in superfluid helium," Proc. Natl. Acad. Sci. 105(37), 13707-13710 (2008).

[80] Abo-Shaeer, J. R., Raman, C., Vogels, J. M., and Ketterle, W., "Observation of Vortex Lattices in BoseEinstein Condensates," Science 292(5516), 476-479 (2001).

[81] Anderson, B. P., Haljan, P. C., Wieman, C. E., and Cornell, E. A., "Vortex Precession in Bose-Einstein Condensates: Observations with Filled and Empty Cores," Phys. Rev. Lett. 85(14), 2857-2860 (2000).

[82] Yao, A. M. and Padgett, M. J., "Orbital angular momentum: origins, behavior and applications," Adv. Opt. Photonics 3(2), 161 (2011).

[83] Franke-Arnold, S., Allen, L., and Padgett, M., "Advances in optical angular momentum," Laser Photon. Rev. 2(4), 299-313 (2008).

[84] Molina-Terriza, G., Torres, J. P., and Torner, L., "Twisted photons," Nat. Phys. 3(5), 305-310 (2007).

[85] Uchida, M. and Tonomura, A., "Generation of electron beams carrying orbital angular momentum," $N a$ ture 464(7289), 737-739 (2010).

[86] McMorran, B. J., Agrawal, A., Anderson, I. M., Herzing, A. A., Lezec, H. J., McClelland, J. J., and Unguris, J., "Electron Vortex Beams with High Quanta of Orbital Angular Momentum," Science 331(6014), 192-195 (2011).

[87] Grillo, V., Gazzadi, G. C., Mafakheri, E., Frabboni, S., Karimi, E., and Boyd, R. W., "Holographic Generation of Highly Twisted Electron Beams," Phys. Rev. Lett. 114(3) (2015).

[88] Lagoudakis, K. G., Ostatnický, T., Kavokin, A. V., Rubo, Y. G., André, R., and Deveaud-Plédran, B., "Observation of half-quantum vortices in an exciton-polariton condensate," Science 326(5955), 974-976 (2009).

[89] Rubo, Y., "Half vortices in exciton polariton condensates," Phys. Rev. Lett. 99(10), 106401 (2007).

[90] Manni, F., Lagoudakis, K., and Liew, T. C. H., "Dissociation dynamics of singly charged vortices into half-quantum vortex pairs," Nat. Commun. 3, 1309 (2012).

[91] Krizhanovskii, D. N., Whittaker, D. M., Bradley, R. A., Guda, K., Sarkar, D., Sanvitto, D., Viña, L., Cerda, E., Santos, P., Biermann, K., Hey, R., and Skolnick, M. S., "Effect of interactions on vortices in a nonequilibrium polariton condensate," Phys. Rev. Lett. 104, 126402 (2010).

[92] Voronova, N. S. and Lozovik, Y. E., "Excitons in cores of exciton-polariton vortices," Phys. Rev. B 86, 195305 (2012). 
[93] Boulier, T., Terças, H., Solnyshkov, D. D., Glorieux, Q., Giacobino, E., Malpuech, G., and Bramati, A., "Vortex Chain in a Resonantly Pumped Polariton Superfluid," Sci. Rep. 5 (2015).

[94] Dufferwiel, S., Li, F., Cancellieri, E., Giriunas, L., Trichet, A. A. P., Whittaker, D. M., Walker, P. M., Fras, F., Clarke, E., Smith, J. M., Skolnick, M. S., and Krizhanovskii, D. N., "Spin Textures of Exciton-Polaritons in a Tunable Microcavity with Large TE-TM Splitting," Phys. Rev. Lett. 115, 246401 (2015).

[95] Liu, G., Snoke, D. W., Daley, A., Pfeiffer, L. N., and West, K., "A new type of half-quantum circulation in a macroscopic polariton spinor ring condensate," Proc. Natl. Acad. Sci. 112(9), 2676-2681 (2015).

[96] Nagali, E., Sansoni, L., Sciarrino, F., De Martini, F., Marrucci, L., Piccirillo, B., Karimi, E., and Santamato, E., "Optimal quantum cloning of orbital angular momentum photon qubits through Hong-Ou-Mandel coalescence," Nat. Photon. 3(12), 720-723 (2009).

[97] Leach, J., Jack, B., Romero, J., Jha, A. K., Yao, A. M., Franke-Arnold, S., Ireland, D. G., Boyd, R. W., Barnett, S. M., and Padgett, M. J., "Quantum Correlations in Optical Angle-Orbital Angular Momentum Variables," Science 329(5992), 662-665 (2010).

[98] Yan, Y., Xie, G., Lavery, M. P. J., Huang, H., Ahmed, N., Bao, C., Ren, Y., Cao, Y., Li, L., Zhao, Z., Molisch, A. F., Tur, M., Padgett, M. J., and Willner, A. E., "High-capacity millimetre-wave communications with orbital angular momentum multiplexing," Nat. Commun. 5, 4876 (2014).

[99] Paterson, C., "Atmospheric Turbulence and Orbital Angular Momentum of Single Photons for Optical Communication," Phys. Rev. Lett. 94(15) (2005).

[100] Krenn, M., Handsteiner, J., Fink, M., Fickler, R., Ursin, R., Malik, M., and Zeilinger, A., "Twisted light transmission over 143 km," Proc. Natl. Acad. Sci. 113(48), 13648-13653 (2016).

[101] Bouchard, F., De Leon, I., Schulz, S. A., Upham, J., Karimi, E., and Boyd, R. W., "Optical spin-to-orbital angular momentum conversion in ultra-thin metasurfaces with arbitrary topological charges," Appl. Phys. Lett. 105(10), 101905 (2014).

[102] Naidoo, D., Roux, F. S., Dudley, A., Litvin, I., Piccirillo, B., Marrucci, L., and Forbes, A., "Controlled generation of higher-order Poincar sphere beams from a laser," Nat. Photon. 10(5), 327-332 (2016).

[103] Wang, J., Yang, J.-Y., Fazal, I. M., Ahmed, N., Yan, Y., Huang, H., Ren, Y., Yue, Y., Dolinar, S., Tur, M., and Willner, A. E., "Terabit free-space data transmission employing orbital angular momentum multiplexing," Nat. Photon. 6(7), 488-496 (2012).

[104] Bozinovic, N., Yue, Y., Ren, Y., Tur, M., Kristensen, P., Huang, H., Willner, A. E., and Ramachandran, S., "Terabit-Scale Orbital Angular Momentum Mode Division Multiplexing in Fibers," Science 340(6140), 1545-1548 (2013).

[105] Karimi, E., Marrucci, L., de Lisio, C., and Santamato, E., "Time-division multiplexing of the orbital angular momentum of light," Opt. Lett. 37(2), 127 (2012).

[106] Picón, A., Mompart, J., Vázquez de Aldana, J. R., Plaja, L., Calvo, G. F., and Roso, L., "Photoionization with orbital angular momentum beams," Opt. Express 18(4), 3660 (2010).

[107] Sigurdsson, H., Egorov, O. A., Ma, X., Shelykh, I. A., and Liew, T. C. H., "Information processing with topologically protected vortex memories in exciton-polariton condensates," Phys. Rev. B 90, 014504 (2014).

[108] Franchetti, G., Berloff, N. G., and Baumberg, J. J., "Exploiting quantum coherence of polaritons for ultra sensitive detectors," arXiv:1210.1187 [cond-mat] (2012). arXiv: 1210.1187.

[109] Pitaevskii, L. and Stringari, S., [Bose-Einstein Condensation], Oxford University Press (2003). 